Published as: Vanclooster, A., Van de Weghe, N., Fack, V., \& De Maeyer, P. (2014). Comparing indoor and outdoor network models for automatically calculating turns. Journal of Location Based Services, vol. 8 (3): 148-165.

\title{
Comparing Indoor and Outdoor Network Models for Automatically
}

\section{Calculating Turns}

Ann Vanclooster ${ }^{\mathrm{a}, *}$, Nico Van de Weghe ${ }^{\mathrm{a}}$, Veerle Fack ${ }^{\mathrm{b}}$, Philippe De Maeyer $^{\mathrm{a}}$

${ }^{a}$ Department of Geography, Ghent University, Krijgslaan 281 (S8)-WE12, 9000 Ghent, Belgium

${ }^{b}$ Department of Applied Mathematics and Computer Science, Ghent University, Krijgslaan 281 (S9)-WE02, 9000 Ghent, Belgium

\{Ann.Vanclooster; Nico.VandeWeghe; Veerle.Fack; Philippe.DeMaeyer\}@Ugent.be

*Corresponding author: Ann Vanclooster; Krijgslaan 281 (S8)-WE12;

Ann.Vanclooster@Ugent.be; Tel: 0032926447 27; Fax: 003292644985

Acknowledgements: This work was supported by the Flanders Research Foundation (FWO-Vlaanderen) under grant number $1123410 \mathrm{~N}$. 


\title{
Comparing Indoor and Outdoor Network Models for Automatically Calculating Turns
}

\begin{abstract}
The goal of this paper is to compare several indoor and outdoor network models for wayfinding, on their suitability for automatically calculating turns. Automatic turn calculations are of relevance in providing improved cognitive algorithms for route guidance, as it has been widely recognized that routes with minimal angular deviations are easier to follow. It is demonstrated that the currently available indoor network models not allow accurate calculation of the number of turns along a path, while the common outdoor route networks do. This discrepancy is found to be rooted in an inconsistent definition of indoor decision nodes which in turn is linked to the inherent differences in space structure between indoor and outdoor environments. Additionally, it is proven that these also have a major influence on the generation of accurate indoor route instructions.

Recommendations for future research within the context of both turn calculations and verbalizations of directional changes are made, as well as in the broader context of indoor spatial analyses.
\end{abstract}

Keywords: network; turn; navigation

\section{Introduction and background}

According to Montello (2005), as long as people have to decide where to go and how to get there, navigation will remain one of the fundamental behavioural problems for human cognition. Navigation processes are said to consist of both locomotion and wayfinding components (Montello 2005). Wayfinding is thereby the process of determining and following a route between origin and destination and is often guided by external aids (Golledge 1999). In the context of this paper, we focus on these guidance aids, improving users' wayfinding experiences, and not on the cognitive act of wayfinding itself. The setting for our research is limited to indoor spaces as research on indoor environments has repeatedly demonstrated the challenges of successfully performing wayfinding tasks in complex three-dimensional spaces (e.g. disorientation 
after vertical travel, less visual routing aid, deficient cognitive map creation) (Hölscher et al. 2009).

Even though wayfinding aids for indoor spaces have gained an enormous amount of interest over the last decade, indoor algorithmic support is still mostly confined to common shortest path algorithms (Vanclooster et al. 2014). In outdoor environments, a set of more 'cognitive' algorithms has specifically been created to deal with wayfinding challenges by providing routes that are more intuitive to follow and more adhering to how people describe paths to unfamiliar users. Several of those algorithms rely on a minimization of number of turns as main cost heuristic (e.g. fewest turns path algorithm, simplest path algorithm). Indeed, turn minimization has been recognized as an important route selection criterion, next to distance and time (Golledge 1995). Also, routes of minimal deviations are often perceived more optimal and comfortable (Winter 2002). Providing these comfortable and easy to follow routes, is even more important indoors than outdoors, as external cues and extrinsic points of view are less manifest in indoor spaces (Padgitt and Hund 2012). A major part of algorithms with turn minimizations is the automatic calculation of turns. Therefore, the goal of this paper is to examine turn calculations on indoor networks and compare them with known efforts in outdoor space. The following sections give an overview of several turn conceptualizations and definitions. Section 2 and Section 3 demonstrate turn calculations on both outdoor road networks and various indoor space representations. In Section 4, several challenges of the indoor application of turn calculations are discussed in more detail.

\subsection{Turn conceptualizations in wayfinding research}

Over time various definitions and measures for detecting turns have been proposed, embedded on different conceptualizations of space. Most commonly, turn calculations 
are of interest for calculating fewest turns paths minimizing the number of directional changes and this using a route graph (Hillier and Iida, 2005). The simplest path algorithm extends this thought as it calculates paths with a minimal route description complexity based on the required amount of information at each intersection. Although simplest path algorithms exist under multiple variants (Mark 1986, Duckham and Kulik 2003, Richter and Duckham 2008), all of them attach a larger cost when dealing with turns. Winter (2002) from his part proposed a line graph to describe turns as edge-edge relationships in response to the common more costly approaches of splitting up graphs in multiple nodes or adding turn penalty tables. Since nowhere is mentioned what exactly is considered a turn, it can only be assumed from the construction rules of the line graph that every outdoor intersection gives occasion to turns. On the other hand, Jiang and Liu (2010) compute fewest turns paths based on a natural routes concept, i.e. where various street edges are merged into a single road. In this case, not every junction is considered a decision point and turns are only counted when changing from one natural road to another, not the directional changes within a natural road.

Space Syntax community presents a highly different view on space structures. One of their conceptualizations of space is the axial map, i.e. a graph of axial lines representing visibility relationships by drawing the fewest longest lines of sight which traverse all convex spaces (Turner et al. 2001). On this axial map, a spatial integration measure can be calculated, quantifying the number of turns to reach all street segments. As such, it forms a measure of the cognitive complexity of reaching a street and is found to predict pedestrian usage (Turner et al. 2001). The connectivity relationship present in the graph topology models in this case turns as a visual transition instead of the pure connectivity of roads and edges in previously discussed road graphs. 


\subsection{Definition of a turn}

In general, a turn can be defined as a directional change from a reference line (Cambridge Dictionary http://dictionary.cambridge.org/dictionary/british/turn_10). The angle is a central point in this definition, consisting of the corner between two distinct rays issuing from the same vertex. In case of navigation systems and concurrent route instructions, not every change of direction has to be labelled turn. Evidence has shown that some turns are more important to humans than others (Turner 2001). However, there is no agreement on which angles form the boundary for deciding the significance of a directional change. For example, Mark (1986) describes in his simplest path algorithm that an angular change above some threshold incurs a maximal turn cost of 9 . However, the threshold itself has not been mentioned. In more recent wayfinding literature, a turn is defined as a decision to deviate from the straight ahead by more than

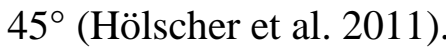

The definition of a turn is also tightly linked to the user's perception on making a significant change in direction, which in turn is connected to how people verbalize navigational paths. Route instruction verbalization is characterized by three main components: (1) structure of decision point, (2) the action itself (directional change or not), and (3) salient features (Klippel et al. 2012). To model the intended action at intersections, different directional models have been developed over time. For example, Klippel et al. (2005) present an eight-direction model with each sector having an increment of $45^{\circ}$ in the prototypical directions, which has been confirmed in behavioural experiments to include all elements relevant for human direction giving at intersections in city street networks.

The authors decide to concur with this idea and will describe a turn as any directional change deviating from the straight ahead by an angle of $45^{\circ}$ or more. Obviously, there are possibilities to alter this threshold and calculate its impact on the 
results of the number of turns over various algorithmic tests. In this paper, turns are only counted at intersections where path alternatives were available and a decision had to be made. Although in future work, this can be extended to include all types of turns and curvature.

\subsection{Algorithm to automatically calculate the number of turns}

To automatically determine the exact number of turns on a path, it is required to calculate each angle created by three consecutive nodes in the path. One of the alternatives to measuring the size of angle utilizes the gradient, i.e. the grade of a slope, which is equal to the tangent of the angle. As such, in (Figure 1), an algorithm is presented that calculates the angle between two connected edges by using the $\mathrm{x}$ - and $\mathrm{y}$ coordinates of the nodes that form the start and end points of the intersecting lines.

(Figure 2) visualizes the various components used in the algorithm.

<Insert Figure 1 about here>

$<$ Insert Figure 2 about here>

Note that in case of dealing with vertical connectors in 3D indoor space (e.g. staircases or elevators), the slopes would have to be calculated in the vertical plane. Also, depending on the type of staircase and the accuracy with which the network describes the inner complexity of the object, additional turns will have to be calculated on intermediate levels, coinciding with the curvature of the path (Stoffel et al. 2008).

\section{Turn calculation on outdoor network models}

As mentioned in Section 1.1, several examples of algorithms with turn minimization in outdoor environments have been proposed, largely based on traditional route graphs. In this section, we use such route graphs to calculate turns with the coordinates of the individual nodes as key elements. More specifically, we will review as example of an 
outdoor network the automatic turn calculations on the international Geographic Data Files (GDF) standard as this is a well-documented example of outdoor street networks (ISO 2002).

\subsection{GDF standard background}

The GDF standard is an international standard used in outdoor route calculations. It contains multiple classes of typical objects for outdoor navigation, with the 'roads and ferries' data model being the most interesting in this context (Figure 3). The road network can be represented at two different levels of detail (level 1 and level 2). A Road is defined as a Level 2-Feature composed of one, many or no Road Elements and forms a connection between two Intersections. It serves as the smallest independent unit of a road network at Level 2. A Road Element is defined as a linear section of the earth, designed for vehicular movement. It serves as the smallest, independent unit of the road network at Level 1 and is bounded by Junction Elements (ISO 2002).

<Insert Figure 3 about here>

\subsection{Application of automatic turn calculation algorithm to GDF}

The relationship between Roads, Road Elements and Intersections can adopt various shapes. These situations correspond to the figures 15,16 and 18 in the GDF standard (ISO 2002, p.26).

- Road containing 1 Road Element: a 1-on-1 mapping of the original Road Element (Level 1) to a Road in level 2. (Figure 4-left column)

- Road containing 2 Road Elements: 2 Road Elements can be aggregated into 1 Road on level 2 if each Road Element is a one-way Road and the Road is one single functional unit. (Figure 4-right column) 
- Road containing no Road Elements: all Road Elements are mapped onto either one of the Intersections. (Figure 5)

In the following sections we examine these situations in light of their feasibility to accurately calculate turns using the algorithm in (Figure 1).

First, for Roads with a single Road Element, the example in (Figure 4-left) demonstrates that this network model supports accurate turn calculations. Having a path from $\mathrm{A}$ to $\mathrm{D}$, the angles in nodes $\mathrm{B}$ and $\mathrm{C}$ can be easily calculated with the algorithm in (Figure 1). For example, for the turn angle in B, nodes $\mathrm{A}$ and $\mathrm{C}$ are used respectively as $\mathrm{N}_{\text {start }}$ and $\mathrm{N}_{\text {end. }}$. A perceptive turn zone of $90^{\circ}\left(45^{\circ}\right.$ left and right of the straight ahead) designates all areas that are not considered as turns. In this case, line BC deviates more than $45^{\circ}$ from the straight ahead (line ABD) introducing a (right) turn in node $\mathrm{B}$. The same principle applies for the turn calculation in node $\mathrm{C}$ where a left turn is calculated. <Insert Figure 4 about here>

For Roads containing two Road Elements (Figure 4-right), the example shows a similar situation. However, in this case the intersections on level 2 are split up in multiple junctions on level 1 . This leads to a more intricate turn calculation in node $\mathrm{C}$. Over the entire path, four decision points have to be passed, with node $\mathrm{C}$ consisting of three junctions. In node $\mathrm{C}_{1}$, the wayfinder has to continue his path straight ahead (line $\mathrm{C}_{1} \mathrm{C}_{2}$ forms the extension of line $\mathrm{BC}_{1}$ ), while in node $\mathrm{C}_{2}$ a left turn is calculated (segment $\mathrm{C}_{2} \mathrm{C}_{3}$ is located outside the perceptive turn zone in node $\mathrm{C}_{2}$ ). Finally, in node $\mathrm{C}_{3}$, a continuation of the straight ahead is required and as such no change in the number of turns can be detected. However, the adjoining verbal instructions required to support wayfinding along this path have to be altered; i.e. 'take the second street on the left'. Note that in this case, taking the first street on the left (i.e. going left in node $\mathrm{C}_{1}$ ) will not be allowed due to the directionality of the separate streets. 
In case of Roads with no Road Elements (Figure 5), a path from A to D shows that only one turn (in node B or node $\mathrm{E}$ in a level 2 model) is recorded, which is in line with the expected decision making of a wayfinder. On level 1, the angle made by the segments BCD is precisely located within the perception turn zone. Even if this was not the case, the angle in node $\mathrm{C}$ should never be counted as a turn, as it is not a real decision point but rather a merging point with the main road through node $\mathrm{D}$. The decision to turn right is already made in node $\mathrm{B}$.

<Insert Figure 5 about here>

In conclusion, as most of the movement on roads is quite guided and restricted, the calculation of turns does not induce any problems in common road and intersection situations. Independent of the level of detail at which the roads and intersections are modelled, the node-coordinate based algorithm works as expected for turn calculations on outdoor networks.

\section{Turn calculation on indoor network models}

As research on indoor navigation is still in its early stages, the standardization of indoor network models has not yet reached full maturity. Graphs are, also indoors, the main navigational model fitting the requirements of connectivity. Various network options have so far been proposed, starting from a direct spatial unit representation with adjustments resulting in three main clusters: corridor derivation, cell decomposition and visibility partitioning. (Figure 6) presents two example paths for each of the indoor network representations. Path 1 connects node 1 and node 2 and path 2 links node 3 with node 4. (Table 1) presents the results of the turn calculations using the algorithm in (Figure 1) over the different indoor networks.

<Insert Figure 6 about here>

<Insert Table 1 about here> 


\subsection{Centre-Node Network}

The centre-node network model is the most elementary indoor network possible with a 1-on-1 relationship between geometrical building structure and graph. Each spatial unit is represented by a node at its centre point, with the edges representing the connectivity relationships between the separate spatial units (e.g. Lorenz et al. 2006, Stoffel et al. 2007). This purely topological connectivity model serves as base for several variations, discussed in the next sections, improving some of its shortcomings.

Applying this model to our turn calculation algorithm, results in a non-accurate accounting of turns. The main problem is the non-realistic representation of the actual walking pattern. Given the fact that the intermediate nodes are located in the centre of each spatial unit, the edges connecting those, are theoretically modelled to go through walls. Also, it is not very realistic that a person walking through a building will each time pass by the centre of the room to decide where to go next.

\subsection{Geometric Network Model}

Corridors hold an important position within the internal building structures as they are the major connecting sections that link multiple functional building units. A geometric network model represents those corridors by a sub graph within the total building graph, which results in a more realistic representation of the actual walking pattern indoor. Several options have been developed with the corridor as line structure (e.g. Lee 2004).

Again, a significant miscalculation in the number of turns is visible due to a mismatch between the indoor network and the actual walking pattern. Most often, these mistakes are induced in large open areas which are either modelled (1) by a single node or (2) by multiple nodes in a sub graph, both inducing unrealistic and unnecessary turn behaviour. Node A (on path 1) forms the topologic representation of a spatial unit, in 
this case a quite large room. The created angle using solely this centre node is in this example smaller than our threshold of $45^{\circ}$, not creating a turn while in the actual walking pattern a turn is experienced. Also, because of this unrealistic centre point, the consecutive edges and nodes create further miscalculations. The angle itself is defined by the wrongful modelling (under-modelling of the spatial unit) of the walking pattern. On the other hand, the main mismatch in path 2 occurs around nodes $\mathrm{B}_{1}$ and $\mathrm{B}_{2}$, a corridor subdivided in various sub-nodes according to the SMAT technique (Lee 2004). However, the actual walking pattern ignores this over-modelling of the spatial unit and takes a more direct door-to-door path.

\subsection{Cell-decomposed Model}

In a cell-decomposed model, large open areas, generally modelled by a single node, are subdivided into multiple cells portraying more accurately the internal room complexity, with each individual cell modelled by a single node. Having a more detailed representation of a large open area also creates a closer representation of the actual walking pattern through those areas, with for example avoidance of obstacles and inaccessible areas. The creation of cells can be proposed for several reasons such as room size, concavity and functionality (Lorenz et al. 2006). However, automatic transformation between input floor data and cell creation is currently lacking.

The node-coordinate based turn algorithm returns with the cell-decomposed model a more accurate result than with any of the previous models, as the main room around node $\mathrm{A}$ is subdivided into three cells, labelled $\mathrm{A}_{1}$ to $\mathrm{A}_{3}$. This results in the calculation of a turn in node $A_{3}$, which aligns to the actual walking pattern of a user when traversing this room. However, the main problem still remains on deciding which units should be modelled into multiple cells and how they should be subdivided. 


\subsection{Visibility-based Model}

Modelling unit by unit often does not correspond to the actual walking pattern of users in the building, as humans rely on a more visibility based spatial reasoning. In such a straight door-to-door visibility-based model, all doors (nodes in the graph) are connected with an edge when there is a direct line of sight. For non-immediate visible door nodes, a visibility partitioning (e.g. Stoffel et al. 2007, Zheng et al. 2009) can be performed, creating intermediate nodes.

The results of the turn calculations using a direct door-to-door visibility based network model show that the algorithm not necessarily calculates correct results. The visibility model returns less angles compared to the actual walking pattern because of its immediate door-to-door connections making the user sometimes go in an extremely sharp angle through a door. This model has also no immediate connection with the actual spatial units themselves, losing an important aspect for route instructions as people mostly connect with those spatial units and not with the doors connecting them.

\section{Discussion}

Previous analyses have shown that with current indoor network models and a simple node-coordinate based algorithm, the exact number of turns could not consistently be deducted in indoor spaces. On outdoor networks, the turn calculation results align with the perceptive notion of turns. In this section we go back to the construction theory behind the networks to discover the reasons for these different results and their implications in a broader context.

\subsection{Difference between morphological and decision nodes}

Before delving in into the actual construction rules of network nodes, it is important to establish the difference between decision nodes and morphological nodes. Decision 
nodes can be defined as nodes created at intersections having multiple choices of next possible paths for the user. The opposite is true for morphological nodes inducing a change in direction without facilitating a choice between different paths (i.e. internal curvature). Both types of nodes can be found in outdoor and indoor networks. However, in most cases, only decision nodes are used for calculating routes.

The type of node influences the results of turn calculations. For example, (Figure 4) and (Figure 5) both showed examples where the outdoor network consisted of only decision nodes. However, (Figure 7) demonstrates that outdoor networks can contain strong intermediate curvature between two consecutive intersections. By using only the coordinates of the decision nodes in the turn calculations, no turn is detected in Node 2 (the outgoing edge is located in the $45^{\circ}$ turn zone). However, when taking the last node before and the first node after the intersection (Node 2) into account (in this example Nodes A and B), independent of their type, a turn is accounted for in this intersection, as such coinciding with the actual perception of a left turn. Therefore, the algorithm in (Figure 1) will need to take into account both decision nodes and morphological nodes and always rely on the last node before and the first node after the decision node to base the $45^{\circ}$ threshold area upon. The $45^{\circ}$ threshold area still only applies to decision nodes as turns are only defined in those nodes where a decision is pushed upon the user.

$<$ Insert Figure 7 about here>

This disambiguation between node types and their influence on turn calculations also holds for indoor networks. Both coordinates of the last node before and the first following the indoor decision node have to be used in the algorithm in (Figure 1). As such, a more accurate perception of turns can be calculated, independent of where exactly the nodes are placed in (indoor) open areas. 


\subsection{Decision node creation rules in networks}

Decision points play a pertinent role in the segmentation of route as goal-directed behaviour (e.g. Klippel et al. 2005), since a wayfinder follows route segments to a decision point where a directional choice is made leading to a new route segment. This definition assumes an underlying network structure of space where the crossing of separate branches creates decision points.

In the construction of roads and intersections in the GDF standard, the basic guideline is functionality in terms of car driving. An Intersection is created when the extended sides of the roads overlap, at which two Junctions will be combined into one (Figure 8). If this is not the case, the two Junctions remain as two independent Intersections. An intersection can only occur where a choice between multiple road segments is available and as such a decision is pushed upon the users. The angle for deciding whether turning into a side route is defined as turn, is then modelled in this point following the direction where the wayfinder came from. Since centrelines of roads are quite easily constructed, defining outdoor decision points is fairly straightforward as they coincide with the actual point of decision making.

$<$ Insert Figure 8 about here>

In indoor space, the various networks demonstrate a different creation theorem for indoor decision nodes (Table 2) and is key to the wrongful calculation of turns in indoor environments. Remark that a similar subdivision is made between decision nodes (where the user has to make a choice between multiple directions) and morphological nodes (visualizing the internal curvature).

<Insert Table 2 about here>

The indoor network model closest to the actual walking pattern in terms of decision node criterion is the visibility-based network. This network also returned the 
closest results in terms of turn calculations. Their common concept is the importance of doorways as starting point for decision making. However, the actual walking pattern alters this idea as not necessarily the door opening itself, but locations in front of the door opening itself can disambiguate between possible choices. This is a result of the fact that as humans, we walk in a plane perpendicular to the door opening. Additionally, some choices cannot be made in the door opening itself due to the concavity of rooms, and a point further within the room serves then as decision point.

As the different indoor models rely on various decision node criteria, it might be interesting to draw some parallelisms between the outdoor intersection creation and the indoor equivalent. After all, the outdoor turn calculations completely coincide with the actual perception of turns, while all indoor models return in some way wrongful turn results.

First, an exact copy of the intersection creation from outdoor space (Figure 8) to indoor environments is shown in (Figure 9-left). The idea is that indoor intersections are formed through the crossing of centrelines modelling the various rooms. Intersections can only be formed when two rooms are connected through a doorway. For example, rooms $\mathrm{C}$ and $\mathrm{D}$ are connected through a mutual door and as such their centrelines cross at a point in room D. Even though this network returns good results in terms of turn calculations, the main problem is that the created decision points are not necessarily linked to specific spatial units themselves. For example, although room B has a path through the centre of its unit connecting rooms $\mathrm{A}$ and $\mathrm{D}$, the spatial unit itself is not modelled by a separate node, creating a loose relationship between the network graph and how people actually reason about indoor units. This is also the reason why most indoor networks at this point are built from modelling each spatial unit individually. 
A slightly adjusted model draws centrelines through the actual doorways connecting two rooms (as doors have been proven to be key in the calculation of turns) perpendicular to the plane of the wall where the door is located. The same problem with the disconnected relationship between graph and spatial unit remains, although the graph itself resembles the actual walking pattern more closely. However, in some cases (e.g. room E), the decision point is located outside the space of the spatial unit itself, making it not useful in the automatic calculation of the turns (Figure 9-right). As such the question remains to where exactly the decision point in indoor space should be best located, to be used in turn calculations.

$<$ Insert Figure 9 about here>

\subsection{Influence on verbal route instructions}

There is an inherent link between directional changes detected by measuring the geometrical angle of change in movement and verbal route instructions with which those directional changes can be explained to users.

The generation and analysis of the effectiveness of outdoor route instructions has already experienced a long history within spatial cognition research (e.g. Daniel et al. 2003). More recent are studies examining the different components of why some parts of directions are perceived as being more difficult than others and how this can help in improving automated route guidance systems (Hirtle et al. 2010). Providing and following accurate route instructions in indoor environments are found to be more critical than outdoors (due to less external clues to maintain orientation). It is also more beneficial to know the particular routes than to know what cardinal direction to follow (Padgitt and Hund 2012). However, the following example demonstrates the intricate relationship between route instruction generation and indoor networks. 
Using the visibility-based network (for its relationship to actual walking patterns), the $45^{\circ}$ turn threshold is drawn in the door opening. Every next door opening, located in this zone, is considered as 'straight ahead' from the previous door. For example, in (Figure 10-left), doors B and C are considered straight ahead from door N, while doors A and D require respectively a left and a right turn. However, the area of $45^{\circ}$ turn angle extends indefinitely into the open space area, making doors that are actually requiring a turn, fit in the area of 'straight ahead'. For example, in (Figure 10middle) door A is now considered as being straight ahead from node $\mathrm{N}$, even though it is located at the exact same location in a slightly expanded spatial unit. Note also that again door $\mathrm{C}$ is considered straight ahead, even though it is part of a perpendicular wall on the right side of door $\mathrm{N}$. One could discuss why door $\mathrm{D}$ is considered to be on the right and door $\mathrm{C}$ on the straight ahead of door $\mathrm{N}$, while verbal instructions might distinguish them as 'close right' versus 'far right'. As such, the thresholds distinguishing those verbal descriptors might require a finer granularity in modelling the indoor spatial unit as to map the right description to the actual wayfinding perspective. <Insert Figure 10 about here>

A space subdivision (similar to the cell-decomposition model) could be the solution where the spatial unit is subdivided into smaller areas each being modelled by a single node (Figure 10-right). In this case, the room is subdivided into two cells, making that only door B is in the straight ahead zone, while door A requires a left turn from door $\mathrm{N}$ and doors $\mathrm{C}$ and $\mathrm{D}$ a right turn. In turn, this example highlights a problem of scaling, i.e. to what extent does the space need to be subdivided into smaller sub units to capture the full meaning of the various verbal route instructions and as such also the correct interpretation of directional changes? 
The example in (Figure 10) demonstrates the problematic nature of using indoor networks in the disambiguation of turns and in the generation of route instructions. Additional problems arise when considering the relationship between direction concepts, their directional models and the underlying spatial structure in which the performed action is embedded (Klippel et al. 2012). Indeed, participant's strategies for verbalizing route instructions are found to change along with the complexity of the intersections (Klippel et al. 2012). While angular directions allow some flexibility, i.e., they can be modelled in different sectors (right versus sharp right), the concept for straight seems to be an axis as far as simple intersections are concerned (Klippel et al. 2004). However, this becomes more complex if the action to be instructed takes place (a) at a complex intersection or (b) if competing branches require a disambiguation of the situation.

Route instructions for indoor space have not yet been studied that extensively. To our knowledge, the work of Mast et al. (2012) is one of the only ones touching upon the complexities of indoor verbal route instruction generation. They conclude that generic route instructions are not sufficient as they rely on network representations which are not able to model the indoor spatial complexities. For example, open spaces might not contain any clearly identifiable paths or decision points, making it illogical to impose a network structure. Instead, Rüetshi and Timpf (2005) define the concept of scene spaces with a hierarchical arrangement of objects as opposed to network spaces containing an inherent network structure. Mast and Wolter (2013) use this distinction for a more accurate creation of indoor route instructions. They conclude that even though wayfinding through both space concepts requires the determination of next possible directions, a clear delineation of 'decision points' in scene space is much harder. This is in line with our conclusions made in Section 4.2. However, their work in 
defining improved ways to generate route instructions in scene spaces is still in progress.

\subsection{Concluding remarks}

This discussion has led to the following main conclusions in a more general context of indoor navigation research and indoor Location-Based Services.

First, the mapping of movement to decision nodes in the network is the main challenge, not the calculation of turns themselves. This is due to the inherent differences between indoor and outdoor spaces, more specifically the contrast between the freedom of movement in indoor spaces versus more regulated and restricted movement in outdoor street networks. It can be concluded that not a single indoor network model at this point is all encompassing in dealing with turns. Every network poses new challenges to turn calculations. The visibility-based network might be the closest in modelling walking patterns, as it relies on similar concepts (visibility aspect, decision points in doorways). However, turn calculations are not accurate due to the sharp angles with which some doorways are entered. On the other hand, cell decomposition allows the mapping of spatial units with a finer granularity (which can help for example the accuracy of route instructions) but there is no theorem on the exact size and location of those cells.

Some situations will indeed lead to better results in terms of turn calculations, but this seems more related to the geometry of the spatial units and not necessarily to the network description itself. As such, for more accurate turn calculations, doors form the key element together with treating every spatial unit by itself. At this point, we are developing a network independent algorithm for indoor turn calculations in line with the perceptual notion of directional changes in indoor space instead of trying to come up with a 'perfect' indoor network. 
Second, on top of the already hampered turn calculations, the specificities of indoor spaces pose some additional challenges for the generation of indoor route instructions. Imposing a network-based verbal route instruction creation method on scene space objects impedes the effectiveness of those instructions. However, the practical implementation of scene versus network space into indoor wayfinding and algorithms is not applicable yet and this for several reasons: (1) indoor route instruction creation is still at its infancy with the main problem remaining the definition of scene spaces and the categorization of all possible semantic objects that make up indoor scenes (Mast and Wolter 2013). (2) Aiding wayfinding by providing appropriate algorithms requires selecting paths from a network (Golledge 1999). Algorithms for navigation need a topology of connectivity to run on, which cannot be provided by the strict containment hierarchy present in scene spaces. Network models on the other hand are based on modelling this topologic relationship of connectivity, also indoors. How the network should be structured to capture the requirements for indoor route instructions remains currently still an open question.

Apart from a different theory for indoor route description modelling, the relationship of direction concepts and intersection types indoors is also up for further investigation. We might not have to deal with different types of intersections indoor in the strict sense but might require a vaguer concept. Empirical tests on what is perceived as a turn in different indoor situations could be a first step towards an increased knowledge on the topic. This should be combined with tests on which indoor route instruction accompanies which indoor situation. Indeed, one can compute easily turns, but did the person moving really make a change in direction and did he perceive it as such? 
Although we focused on solutions for indoor turn calculations (and as such facilitating for example the application of fewest turns path algorithm indoors), bringing other algorithms and analytical functions to the indoor world can pose similar challenges. The inherent problem still remains the modelling of indoor areas by networks. Even though indoor environments are open space areas, they are still bounded by multiple impenetrable boundaries (at least for human users in navigation applications). Many data sources assume an 'ideal space', i.e. represented by unbounded homogenous space with Euclidean distances (Okabe and Sugihara 2012). However, ideal space is far from the real world, especially with respect to indoor environments. Indoor analyses have to deal with constraint, non-Euclidean space. While a simple indoor context can get by with a network abstraction, the coarseness of this representation can become inconsistent with more complicated analyses. As shown in Section 3, various options for indoor networks have already been presented. It is however not clear yet what and if there is a perfect indoor network available. Ongoing research on 3D routing using the IndoorGML standard (OGC, 2014) might be a valuable start for further research on determining an improved structure of indoor networks. On the other hand, more research might be required for the development of improved methodologies for indoor analyses tailored to the specificities of indoor spaces (e.g. by extending research on 3D indoor routing based on Indoor GML). A starting point can be the work of Okabe and Sugihara (2012) presenting common analytical concepts adapted to network spaces. Additionally, one can examine the results of these analyses over the various available indoor network options in order to provide a more comprehensive indoor network structure and understand the implications on analytical results. 


\section{Conclusion}

In this paper, the problem of automatic turn calculation on indoor network models was highlighted. Accurate turn calculations are of relevance for a consistent implementation of cognitive algorithms based on minimization of turns as cost heuristic (e.g. fewest turns path, simplest path algorithm). Turn calculations based on a node-coordinate based algorithm were executed in both an example of an outdoor road network and several indoor network models. While in outdoor space, accurate results could be obtained independent of the level of detail, all indoor network options showed aberrations with the actual perception of indoor turns. It was demonstrated that these aberrations were rooted on a different creation of networks and as such also a different underlying meaning and formation of decision points. This is due to the inaccurate modelling of indoor scene spaces by networks which generalize both the required granularity for navigation applications as well as the appropriate modelling of verbal route instructions and directional changes. Therefore, we suggest the development of a network independent algorithm for indoor turn calculations in line with the perceptual notion of directional changes in indoor space. Furthermore, more research is required into the relationship between indoor network structures and the results of indoor analyses.

\section{References}

Daniel, Marie-Paule, Ariane Tom, Elsa Manghi, and Michel Denis. "Testing the Value of Route Directions through Navigational Performance." Spatial Cognition \& Computation 3, no. 4 (2003): 269-89.

Duckham, Matt, and Lars Kulik. "“Simplest" Paths: Automated Route Selection for Navigation." In Spatial Information Theory. Foundations of Geographic Information Science, edited by Walter Kuhn, Michael Worboys and Sabine Timpf. Lecture Notes in Computer Science, 169-85. Heidelberg: Springer, 2003. 
Golledge, Reginald G. "Path Selection and Route Preference in Human Navigation: A Progress Report." Chap. 14 In Spatial Information Theory a Theoretical Basis for GIS, edited by Andrew Frank and Werner Kuhn. Lecture Notes in Computer Science, 207-22. Heidelberg: Springer, 1995.

Golledge, R. G. Wayfinding Behavior, Cognitive Mapping and Other Spatial Processes. Baltimore: The Johns Hopkins University Press, 1999.

Hillier, Bill, and Shinichi Iida. "Network and Psychological Effects in Urban Movement." Chap. 30 In Spatial Information Theory, edited by Anthony G. Cohn and David M. Mark. Lecture Notes in Computer Science, 475-90. Berlin: Springer, 2005.

Hirtle, Stephen, Kai-Florian Richter, Samvith Srinivas, and Robert Firth. "This Is the

Tricky Part: When Directions Become Difficult." Journal of Spatial Information Science, no. 1 (2010): 53-73.

Hölscher, Christoph, Simon J. Büchner, Tobias Meilinger, and Gerhard Strube.

"Adaptivity of Wayfinding Strategies in a Multi-Building Ensemble: The Effects of Spatial Structure, Task Requirements, and Metric Information." Journal of Environmental Psychology 29, no. 2 (2009): 208-19.

Hölscher, Christoph, Thora Tenbrink, and Jan M. Wiener. "Would You Follow Your Own Route Description? Cognitive Strategies in Urban Route Planning.". Cognition 121, no. 2 (2011): 228-47.

ISO. "Iso/Dis 14825 Intelligent Transport Systems - Geographic Data Files (Gdf) Overall Data Specification." In ISO/TC 211 Geographic information/Geomatics, 555: ISO, 2002.

Jiang, Bin, and Xintao Liu. "Automatic Generation of the Axial Lines of Urban Environments to Capture What We Perceive.". International Journal of Geographical Information Science 24, no. 4 (2010): 545-58.

Klippel, Alexander, Carsten Dewey, Markus Knauff, Kai-Florian Richter, Dan R. Montello, Christian Freksa, and Esther-Anna Loeliger. "Direction Concepts in Wayfinding Assistance Systems." Paper presented at the Workshop on Artificial Intelligence in Mobile Systems (AIMS'04), Saarbrücken, 2004.

Klippel, Alexander, Heike Tappe, Lars Kulik, and Paul U. Lee. "Wayfinding Choremes - a Language for Modeling Conceptual Route Knowledge." Journal of Visual Languages \& Computing 16, no. 4 (2005): 311-29. 
Klippel, Alexander, Thora Tenbrink, and Daniel R. Montello. "The Role of Structure and Function in the Conceptualization of Directions." In Motion Encoding in Language and Space, edited by Mila Vulchanova and Emile van der Zee. 10222. Oxford: Oxford University Press, 2012.

Lee, Jiyeong "A Spatial Access-Oriented Implementation of a 3-D Gis Topological Data Model for Urban Entities.". Geoinformatica 8, no. 3 (2004): 237-64. Lorenz, Bernhard, Hans Ohlbach, and Edgar-Philipp Stoffel. "A Hybrid Spatial Model for Representing Indoor Environments." In Web and Wireless Geographical Information Systems, edited by James Carswell and Taro Tezuka. Lecture Notes in Computer Science, 102-12. Heidelberg: Springer, 2006.

Mark, David M. "Automated Route Selection for Navigation." [In English]. IEEE Aerospace and Electronics Systems Magazine 1, no. 9 (1986): 2-55.

Mast, Vivien, Cui Jian, and Desislava Zhekova. "Elaborate Descriptive Information in Indoor Route Instructions." In Proceedings of the 34th Annual Conference of the Cognitive Science Society. Austin (TX): Cognitive Science Society, 2012.

Mast, Vivien, and Diedrich Wolter. "A Probabilistic Framework for Object Descriptions in Indoor Route Instructions." Chap. 11 In Spatial Information Theory, edited by Thora Tenbrink, John Stell, Antony Galton and Zena Wood. Lecture Notes in Computer Science, 185-204. Berlin: Springer, 2013.

Montello, Daniel R. "Navigation." In The Cambridge Handbook of Visuospatial Thinking, edited by P. Shah and A. Miyake. 257-94. New York: Cambridge University Press, 2005.

Okabe, Atsuyuki, and Kōkichi Sugihara. Spatial Analysis Along Networks: Statistical and Computational Methods. Statistics in Practice. John Wiley \& Sons, Ltd, 2012.

Open Geospatial Consortium. "OGC IndoorGML." 90pp: Jiyeong Lee, Ki-Joune Li, Sisi Zlatanova, Thomas Kolbe, Claus Nagel, Thomas Becker, 2014.

Padgitt, Amanda J., and Alycia M. Hund. "How Good Are These Directions? Determining Direction Quality and Wayfinding Efficiency." Journal of Environmental Psychology 32, no. 2 (2012): 164-72.

Richter, Kai-Florian, and Matt Duckham. "Simplest Instructions: Finding Easy-toDescribe Routes for Navigation." In Geographic Information Science, edited by Thomas Cova, Harvey Miller, Kate Beard, Andrew Frank and Michael 
Goodchild. Lecture Notes in Computer Science, 274-89. Heidelberg: Springer, 2008.

Rüetschi, Urs-Jakob, and Sabine Timpf. "Modelling Wayfinding in Public Transport: Network Space and Scene Space." Chap. 2 In Spatial Cognition Iv. Reasoning, Action, Interaction, edited by Christian Freksa, Markus Knauff, Bernd KriegBrückner, Bernhard Nebel and Thomas Barkowsky. Lecture Notes in Computer Science, 24-41. Heidelberg: Springer, 2005.

Stoffel, Edgar-Philipp, Bernhard Lorenz, and Hans J. Ohlbach. "Towards a Semantic Spatial Model for Pedestrian Indoor Navigation." In Advances in Conceptual Modeling - Foundations and Applications. Lecture Notes in Computer Science, 328-37. Berlin: Springer, 2007.

Stoffel, Edgar-Philipp, Korbinian Schoder, and Hans J. Ohlbach. "Applying Hierarchical Graphs to Pedestrian Indoor Navigation." In Proceedings of the 16th ACM SIGSPATIAL international conference on Advances in geographic information systems. Irvine (CA): ACM, 2008.

Turner, Alasdair "Angular Analysis." In 3rd International Space Syntax Symposium, 30.1-30.11. Atlanta (USA), 2001.

Turner, Alasdair, Maria Doxa, David O'Sullivan, and Alan Penn. "From Isovists to Visibility Graphs: A Methodology for the Analysis of Architectural Space.". Environment and planning B, Planning and design 18, no. 1 (2001): 103-21. Vanclooster, Ann, Kristien Ooms, Pepijn Viaene, Veerle Fack, Nico Van de Weghe, and Philippe De Maeyer. "Evaluating Suitability of the Least Risk Path Algorithm to Support Cognitive Wayfinding in Indoor Spaces: An Empirical Study." Applied Geography 53, no. 0 (2014): 128-40.

Winter, Stephan. "Modeling Costs of Turns in Route Planning." Geoinformatica 6, no. 4 (2002): 345-61.

Zheng, Jianghua, Adam Winstanley, Zheng Pan, and Seamus Coveney. "Spatial Characteristics of Walking Areas for Pedestrian Navigation." In 3th International Conference on Multimedia and Ubiquitous Engineering, 452-58. Qingdao, China, 2009. 
Table 1. Comparison between the calculated number of turns using various indoor network structures.

Table 2. Decision node criterion for several indoor networks.

Figure 1. Node-coordinate based algorithm for turn calculations.

Figure 2. Visual explanation of the node-coordinate based algorithm for turn calculations.

Figure 3. Part of the data model 'Roads and Ferries' over various levels of detail.

Figure 4. Turn calculations on a Road with 1 (left) and 2 (right) Road Element(s).

Figure 5. Turn calculations on Roads with no Road Elements.

Figure 6. Overview of several indoor network structures and their influence on turn calculations. (a) Centre-Node Network; (b) Geometric Network Model (GNM) with only room nodes; (c) GNM with room and door nodes; (d) Cell-decomposed Network Model; (e) Visibility-based model; (f) Actual walking pattern.

Figure 7. Morphological and decision nodes in an outdoor road network.

Figure 8. Intersection construction rules in the GDF standard (based on ISO 2002).

Figure 9. Creation of indoor decision nodes at the intersection of the extended doorways.

Figure 10. Doors as decision nodes in indoor space. 